\title{
Developing de novo human artificial chromosomes in embryonic stem cells using HSV-1 amplicon technology
}

\author{
Daniela Moralli • Zoia L. Monaco
}

Published online: 6 February 2015

(C) The Author(s) 2015. This article is published with open access at Springerlink.com

\begin{abstract}
De novo artificial chromosomes expressing genes have been generated in human embryonic stem cells (hESc) and are maintained following differentiation into other cell types. Human artificial chromosomes (HAC) are small, functional, extrachromosomal elements, which behave as normal chromosomes in human cells. De novo HAC are generated following delivery of alpha satellite DNA into target cells. HAC are characterized by high levels of mitotic stability and are used as models to study centromere formation and chromosome organisation. They are successful and effective as gene expression vectors since they remain autonomous and can accommodate larger genes and regulatory regions for long-term expression studies in cells unlike other viral gene delivery vectors currently used. Transferring the essential DNA sequences for HAC formation intact across the cell membrane has been challenging for a number of years. A highly efficient delivery system based on HSV-1 amplicons has been used to target DNA directly to the ES cell nucleus and HAC stably generated in human embryonic stem cells (hESc) at high frequency. HAC were detected using an improved protocol for hESc chromosome harvesting, which consistently produced high-quality metaphase spreads that
\end{abstract}

Responsible Editor: Natalay Kouprina and Vladimir Larionov

D. Moralli

The Wellcome Trust Centre for Human Genetics, University of Oxford,

Roosevelt Drive, Oxford OX3 7BN, UK

Z. L. Monaco $(\bowtie)$

Department of Biomedical Engineering, Tufts University,

4 Colby Street, 02155-6013 Medford, MA, USA

e-mail: zoia.monaco@tufts.edu could routinely detect $\mathrm{HAC}$ in hESc. In tumour cells, the input DNA often integrated in the host chromosomes, but in the host ES genome, it remained intact. The hESc containing the HAC formed embryoid bodies, generated teratoma in mice, and differentiated into neuronal cells where the HAC were maintained. The HAC structure and chromatin composition was similar to the endogenous hESc chromosomes. This review will discuss the technological advances in HAC vector delivery using HSV-1 amplicons and the improvements in the identification of de novo HAC in hESc.

Keywords gene therapy · gene expression · viral replication $\cdot$ viral vector
Abbreviations
AAV Adeno-associated virus
FISH Fluorescence in situ hybridization
HAC Human artificial chromosome
hESc Human embryonic stem cells
HSV-1 Herpes simplex virus type 1
HPRT Hypoxanthine phosphoribosyltransferase

\section{Introduction}

De novo human artificial chromosomes (HAC) are small, extrachromosomal elements that contain a functional centromere, enabling their correct replication and segregation as stable normal chromosomes in human cells (Kouprina et al. 2014; Grimes and Monaco 2005), together with the endogenous chromosomes. De novo HAC are generated by introducing specific 
sequences such as $\alpha$-satellite (alphoid) DNA into eukaryotic cells. The essential requirement for a functional centromere formation in HAC is $\alpha$-satellite DNA, containing higher-order repeat sequences and a centromere protein B binding sequence (CENP-B box) (Bergmann et al. 2012).

By mechanisms not yet elucidated, the cell is able to recognise the transgenic DNA as centromeric and seed the deposition of specific protein/epigenetic markers on the exogenous molecules, leading to the formation of a fully active centromere, thus transforming the episomal vector into a full-fledged artificial chromosome (Grimes and Monaco 2005). In human cells, autonomous HAC are characterised by high mitotic stability (Grimes and Monaco 2005; Moralli et al. 2009), unlike other viral vectors which integrate into the host chromosome and may result in insertional mutagenesis (Baum 2007). As there is no limit on the size of the DNA fragments that can be incorporated into HAC, they represent ideal vectors for the delivery of large genomic DNA regions containing genes and their corresponding regulatory elements. HAC containing the entire human genomic hypoxanthine phosphoribosyltransferase (HPRT) region fully complemented the HPRT deficiency in cultured human fibrosarcoma HT1080 cells (Mejía et al. 2001). The de novo HAC structure was relatively simple compared to normal chromosomes (Mejía et al. 2001; Moralli et al. 2009, 2013) and allowed easy characterisation of their composition and chromatin environment.

Known methods for the delivery of large pieces of DNA are inefficient, often resulting in DNA shearing and degradation, which is a major obstacle in developing a HAC expression system in different cell types. Current methods of introducing DNA into cells include the use of lipofecting agents, electroporation and viral and non-viral vectors. Each of these methods shares the problem that only small pieces of DNA can be put into cells without the DNA being damaged. Large DNA fragments have very low transfection efficiencies or break up when entering cells using current methods. This has made it very difficult in the past to get sufficiently large pieces of DNA into cells to form a HAC. In order to use a HAC for gene therapy, the efficiency of delivery of existing methods needs to be improved.

Most gene expression studies in human embryonic stem cells (hESc) utilise lentiviral, adenoviral and adeno-associated (AAV) viral vectors for gene delivery. However, lentiviral vectors integrate randomly at multiple sites within the host genome leading to insertional mutagenesis, and although adenoviral vectors remain episomal, silencing post-transduction may occur. Another disadvantage is that the capacity of AAV and lentiviral vectors is limited to approximately 5 and $10 \mathrm{~kb}$ of DNA, respectively.

The herpes simplex virus type 1 (HSV-1)-based amplicon system offers enormous potential as a versatile tool for gene delivery and has several unique features that distinguish it from rest of the viral vector systems and chemical-mediated gene transfer methods. It can accommodate and deliver up to $152 \mathrm{~kb}$ of exogenous DNA into the mammalian cells (Saeki et al. 2003; Wade-Martins et al. 2001). Construction of the amplicon DNA vector is simple and flexible. The amplicon DNA does not integrate into the host chromosomes, thus reducing the risk of insertional mutagenesis. The headto-tail, rolling-circle DNA replication mechanism of HSV-1 also allows amplification of amplicon plasmid as concatemers leading to increased copy number of transgenes and consequently their expression. Most genes in the HSV-1 viral genome are not essential for virus replication and therefore, can be deleted without affecting the virus production from the cultured cells. The lack of viral genes and the availability of amplicon production in a helper virus-free manner results in limited immunogenic and cytotoxic effects. Most importantly, the HSV-1 amplicon can transduce a wide variety of cell types across a wide range of species, including dividing and quiescent cells.

\section{Generating HSV-1 amplicons}

The HAC vectors are firstly retrofitted with the HSV-1 origin of replication and packaging signal (Fig. 1). Infectious particles are then produced by cotransfecting, into the permissive cells such as African green monkey kidney Vero 2-2 cells, (a) the desired amplicon DNA (containing the transgene, the HSV pac signal and HSV oriS), (b) the fHSV $\Delta$ pac $\Delta 270+$ vector containing the complete HSV-1 genome save for the deletion of the pac sequence and the essential gene ICP27, and (c) an ICP27 expressing plasmid such as pEBHICP27 (Saeki et al. 2003) (Fig. 2).

Helper functions for the amplification and packaging/cleavage of amplicon DNA into HSV-1 virions are supplied in trans by fHSV $\Delta$ pac $\Delta 270+$ and pEBHICP27. However, fHSV $\Delta$ pac $\Delta 270+$ itself cannot be packaged because it lacks the packaging signal, 
Fig. 1 Assembly of HSV-1 amplicon HAC vectors. A large BAC, carrying alpha satellite and/ or transgenes, is retrofitted with the HSV-1 necessary elements via LoxP-Cre recombination with a smaller plasmid carrying the HSV-1 origin of replication and packaging signal

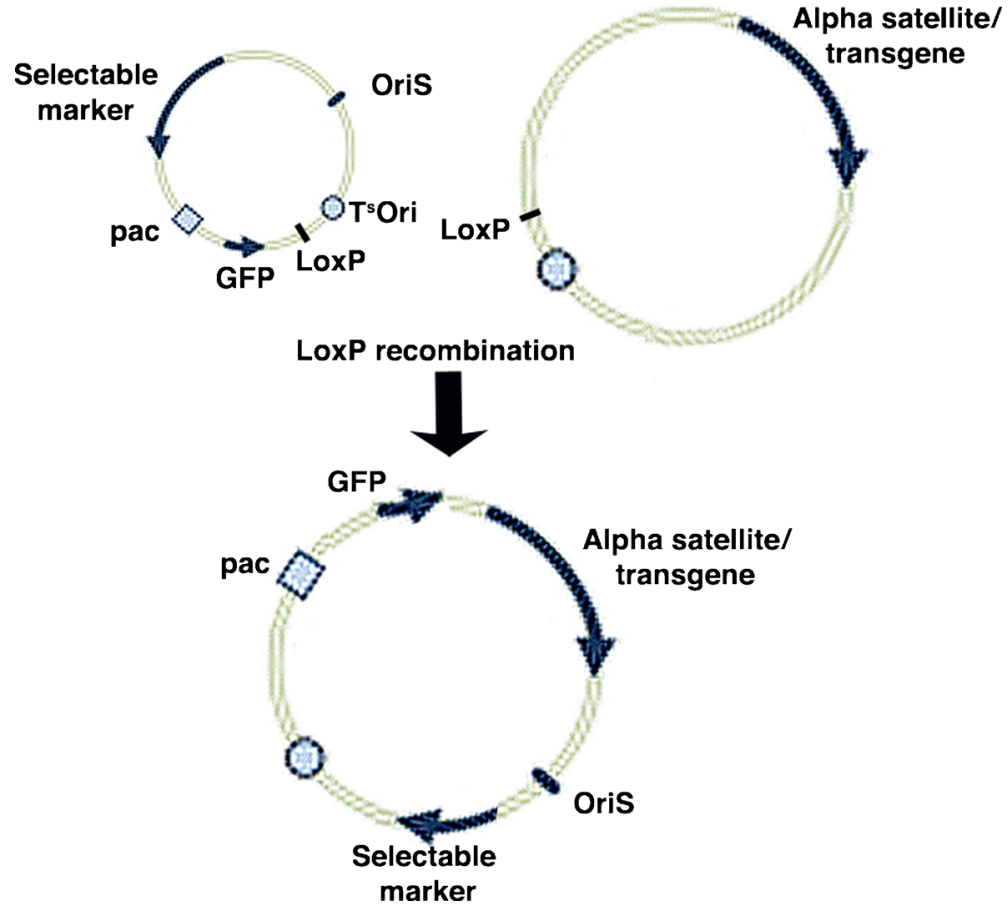

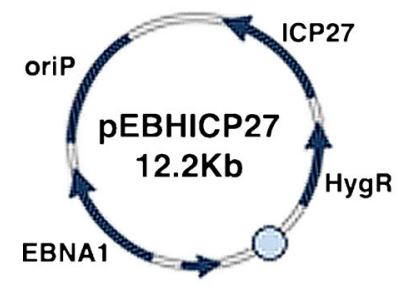
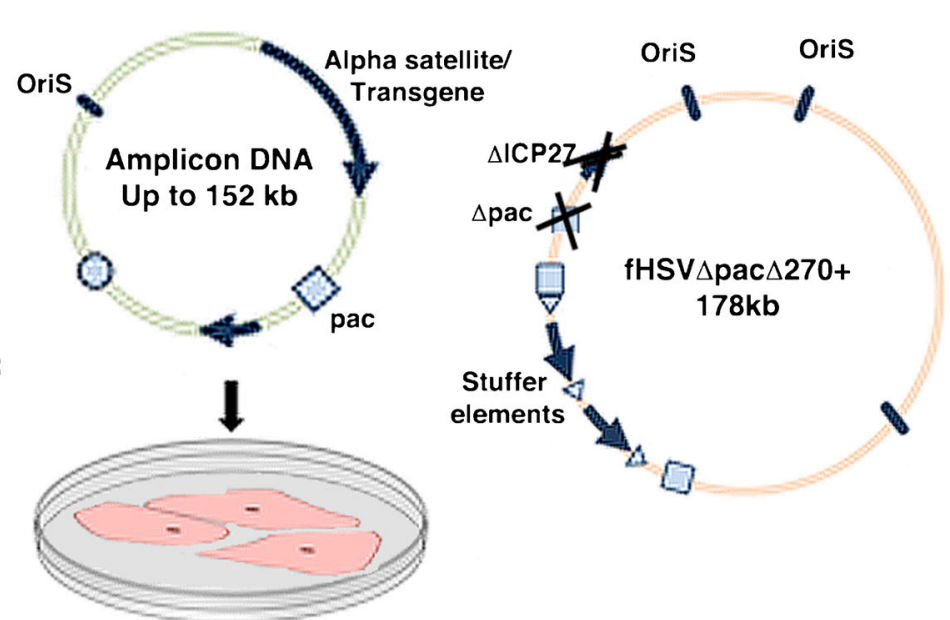

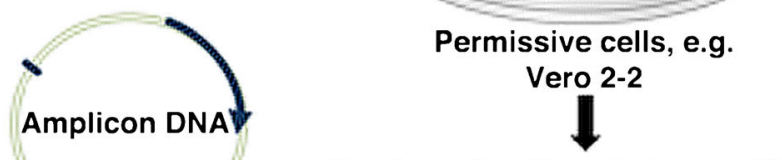

Head to tail rolling circle type DNA replication

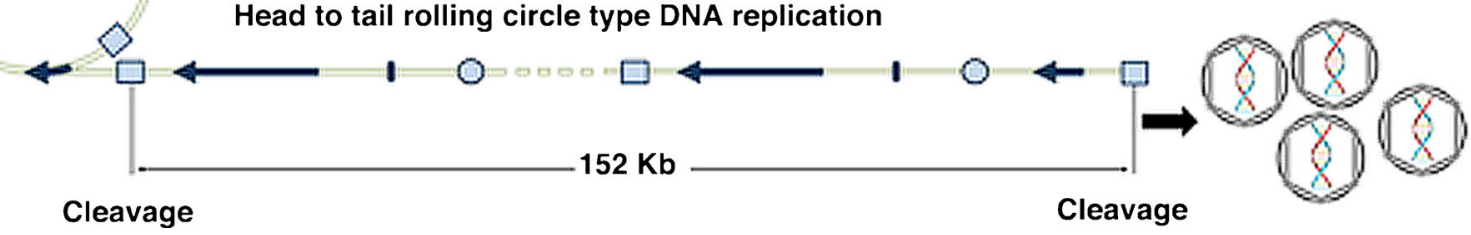

Fig. 2 Production of infectious particles. The HSV-1 amplicon HAC vector is transfected into helper cells along with the whole HSV-1 genome (lacking the ICP27 gene and the packaging signal) and a small plasmid expressing the essential HSV-1 protein ICP27. The cell assembles the HSV-1 capsids and packages the HSV-1 amplicon $\mathrm{HAC}$ vector 
the ICP27 gene and HSV-1 origin of replication. Moreover, its size has been increased by the addition of stuffer elements so that the whole construct no longer fits into the HSV virion, thus further reducing the risk of generating replication-competent helper viruses. The HSV-1 amplicon system, therefore, provides a safe and effective mechanism for gene transfer, as amplicons infect the target cells, but lack the components for generating the wild-type virus.

The HSV-1 amplicon DNA can accommodate one or more transgene cassettes as long as its total size does not exceed $\sim 152 \mathrm{~kb}$. Upon provision of HSV helper functions in trans, the amplicon plasmid undergoes head-totail rolling circle replication and results in concatemers of DNA leading to a total size of $\sim 152 \mathrm{~kb}$ which is then cleaved at the pac signal and packaged into HSV virions (Fig. 1).

The infectious particles are then used to transduce the target cell. The transgenic material is carried directly to the nucleus, intact (Saeki et al. 2003; Wade-Martins et al. 2001). This feature of the HSV-1 amplicon delivery system is especially important for the generation of human artificial chromosomes and has allowed us to obtain direct HAC formation in human embryonic stem cells for the first time.

\section{Establishing HAC in human embryonic stem cells}

The generation of HAC in hESc and iPS cells is an essential step to gene expression and gene therapy studies. Cells obtained from a patient may be reprogrammed to form iPS cells, then modified ex vivo with a HAC vector containing the desired gene and reintroduced into the patient. hESc could be differentiated into immunoglobulin-producing cells, which could prove useful in vaccine production. The HAC generated in our laboratory and others, have to date been obtained in tumour-derived or immortalised cell lines, such as the human fibrosarcoma cell line HT1080. Before HAC can become fully viable vectors for gene expression studies and potentially gene therapy, it is necessary to study their behaviour in cells that have normal genetic backgrounds such as hESc that can be propagated indefinitely in vitro and can be differentiated in a range of different cell types.

In our study, we assembled HSV-1 amplicon technology HAC input vectors carrying reporter genes (Mandegar et al. 2011). We routinely obtained infectious particles suspensions with titres of $10^{8}$ amplicon $/ \mathrm{ml}$. The vectors were delivered to the hESc cell lines HUES-2 and HUES-10 (Cowan et al. 2004) and the results compared to similar experiments in the human fibrosarcoma HT1080 cells, which are highly efficient at HAC formation (Grimes and Monaco 2005). The transduction efficiency using HSV-1 amplicons is high, ranging from 27 to $40 \%$ in hESc (Mandegar et al. 2011) and up to $70 \%$ in HT1080 cells (Moralli et al. 2006). Following G418 selection, several independent clones can be obtained from each vector in both hESc and HT1080 cells.

\section{An improved technique for analysing embryonic stem cells by FISH}

In standard HAC generation experiments, clones are expanded to at least the $10-\mathrm{cm}$ dish stage, before chromosomes are harvested and subjected to fluorescence in situ hybridization (FISH) to determine if the cells contain HACs or integrations. Thus, the karyotypic analysis of a large number of hESc clones can be very timeconsuming and expensive. For this reason, the development of an improved harvesting technique, which allows to obtain high-quality metaphase spreads from sixwell dishes, proved especially useful for our studies (Moralli et al. 2011). This method relies on overnight incubation with a low dose of nocodazole as synchronizing agent. This increases the mitotic index by tenfold, while maintaining a good chromosome structure. The cells are swelled by treatment in a buffered hypotonic, which dramatically improves chromosome spreading. The quality of the resulting metaphase suspension is such that they can be easily analysed by FISH or MFISH (Fig. 3).

\section{Characterisation of HAC in hESc}

In our studies, hESc clones were analysed by FISH with HAC-specific probes (Fig. 4) to determine if the input vector had integrated randomly into the host cell genome or if had formed a HAC able to segregate independently. While in HT1080, the majority of clones contained integrations and HAC, whereas in the hESc clones, the input DNA had formed HAC in a large percentage of cells and there was no integrated DNA present (Mandegar et al. 2011). 

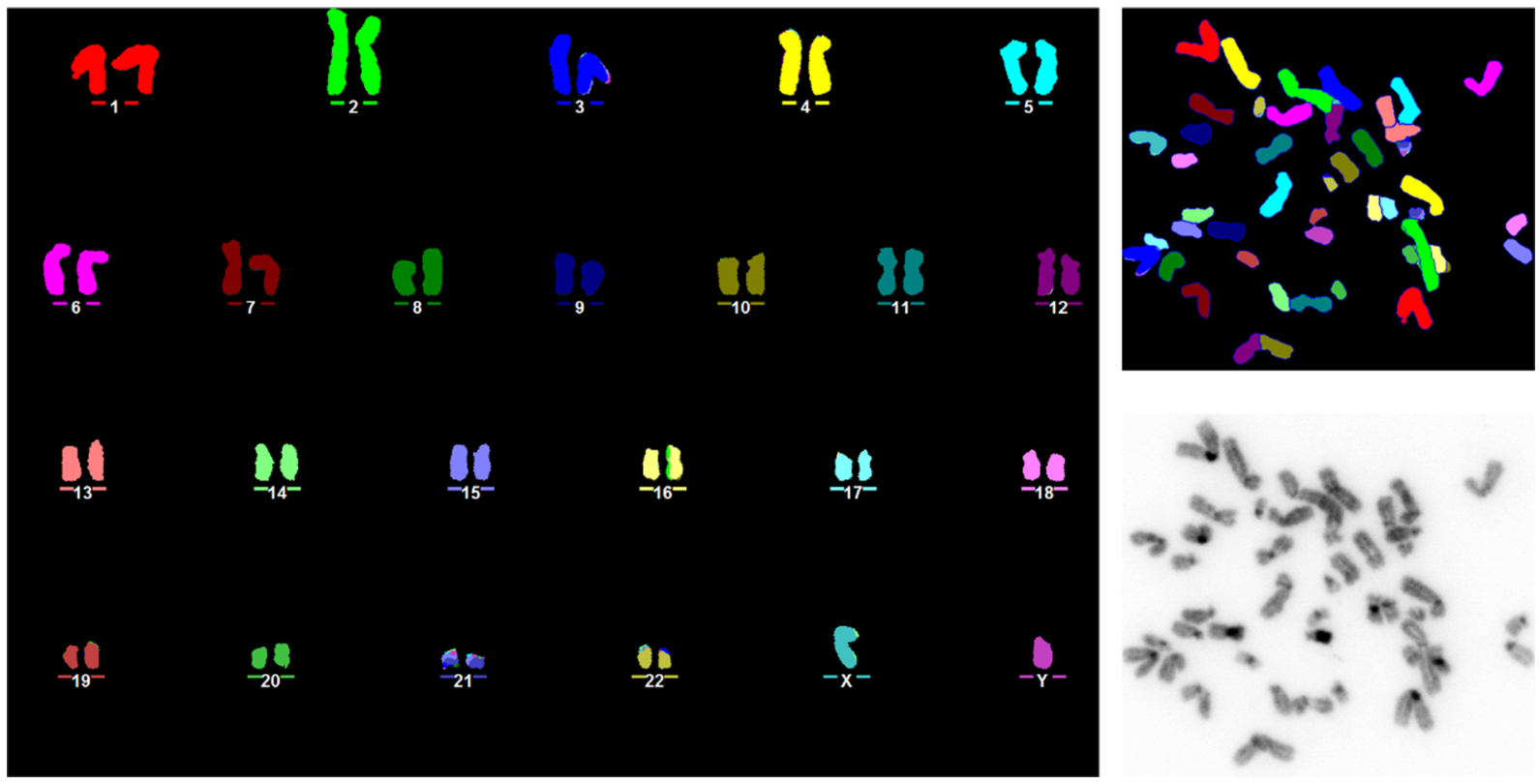

Fig. 3 MFISH on HUES10 hESc metaphase spread, obtained with an improved chromosome harvesting procedure

The analysis of the hESc HAC with antibodies against essential centromeric proteins (CENP C) showed that all the HAC had formed a true centromere. When grown in the absence of selection, the HAC were mitotically stable, displaying a

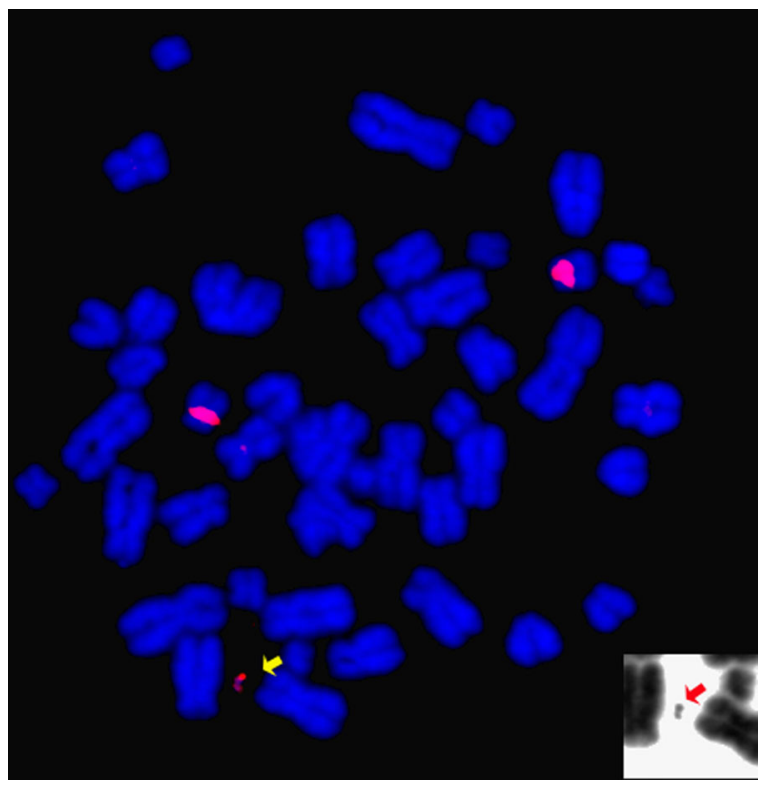

Fig. 4 FISH on HAC containing hESc. The metaphase spread has been hybridised with a 17 alpha satellite probe (red signal) identifying the HAC (arrow), along with the endogenous chromosomes 17. The small inset show the DAPI staining of the HAC, as a black and white picture daily loss rate of $0.03-0.24 \%$. Furthermore, immunostaining and RT-PCR analysis of pluripotency markers (Oct4, Sox2, Nanog, TRA-1-60) indicated that the cells derived from the HAC clones remained pluripotent (Mandegar et al. 2011). The pluripotency of the cells was further confirmed following directed differentiation of HACcontaining cells into neuronal type cells and the development of teratomas with the three germ layers following the formation assay. Importantly, the HAC were maintained in the neuronal cells following differentiation, as shown by FISH with specific probes. Although showing some variability, HAC gene expression was sustained over time. Compared to transfection, the HSV-1 amplicon provided highly efficient delivery of small and large DNA segments to HUES-2 cells, and neither transfection nor the HSV-1 amplicon technique was toxic to HUES-2 cells, as shown by measuring the average growth rate post-transduction (Mandegar et al. 2011).

\section{Conclusion}

The HSV-1 amplicon technology is a highly efficient delivery method based on transferring exogenous DNA packaged in HSV-1 amplicons into cells. The amplicons efficiently deliver large DNA containing genes within high-capacity human artificial chromosome vectors into 
human embryonic stem cells and generate gene expressing HAC in hESc cell lines. The HAC are stable and sustain long-term gene expression. The lack of integrated DNA in hESc following HSV-1 delivery compared to integrated DNA following delivery in cultured cells is a significant difference and will lead to further work on understanding the mechanism of HAC generation in human cells. The differences in genome stability between stem and tumour cells indicate that HAC will be important in gene therapy strategies for monitoring chromosome stability in different cell types. HAC vectors are a viable alternative to gene delivery with viral vectors in hESc with the aim of developing a HACbased system for the delivery of therapeutic genes regulated with tissue-specific promoters to human target cells for ex vivo treatment. The development of ex vivo strategies for gene therapy of inherited genetic disorders and addressing problems in the areas of cancer, aging and metabolic disorders will be key targets for HAC therapy.

Acknowledgments We thank Dr. Mohammad A. Mandegar and Dr. Suhail Khoja for help with the figures. Support is from National Institutes of Health Grant HH44029 to ZM and DM, and Wellcome Trust Core Award Grant 090532/Z/09/Z.

Open Access This article is distributed under the terms of the Creative Commons Attribution License which permits any use, distribution, and reproduction in any medium, provided the original author(s) and the source are credited.

\section{References}

Baum C (2007) Insertional mutagenesis in gene therapy and stem cell biology. Curr Opin Hematol 14:337-342

Bergmann JH, Martins NM, Larionov V, Masumoto H, Earnshaw WC (2012) HACking the centromere chromatin code: insights from human artificial chromosomes. Chromosome Res 20:505-519

Cowan CA, Klimanskaya I, McMahon J, Atienza J, Witmyer J, Zucker JP, Wang S, Morton CC, McMahon AP, Powers D, Melton DA (2004) Derivation of embryonic stem-cell lines from human blastocysts. N Engl J Med 350:1353-1366

Grimes BR, Monaco ZL (2005) Artificial and engineered chromosomes: developments and prospects for gene therapy. Chromosoma 4:230-241

Kouprina N, Tomilin AN, Masumoto H, Earnshaw WC, Larionov V (2014) Human artificial chromosome-based gene delivery vectors for biomedicine and biotechnology. Expert Opin Drug Deliv 11:517-535

Mandegar MA, Moralli D, Khoja S, Cowley S, Chan DY, Yusuf M, Mukherjee S, Blundell MP, Volpi EV, Thrasher AJ, James W, Monaco ZL (2011) Functional human artificial chromosomes are generated and stably maintained in human embryonic stem cells. Hum Mol Genet 20:2905-2913

Mejía JE, Willmott A, Levy E, Earnshaw WC, Larin Z (2001) Functional complementation of a genetic deficiency with human artificial chromosomes. Am J Hum Genet 69:315326

Moralli D, Simpson KM, Wade-Martins R, Monaco ZL (2006) A novel human artificial chromosome gene expression system using herpes simplex virus type 1 vectors. EMBO Rep 7: 911-918

Moralli D, Chan DY, Jefferson A, Volpi EV, Monaco ZL (2009) HAC stability in murine cells is influenced by nuclear localization and chromatin organization. BMC Cell Biol 10:18

Moralli D, Yusuf M, Mandegar MA, Khoja S, Monaco ZL, Volpi EV (2011) An improved technique for chromosomal analysis of human ES and iPS cells. Stem Cell Rev 7:471-477

Moralli D, Jefferson A, Valeria Volpi E, Larin Monaco Z (2013) Comparative study of artificial chromosome centromeres in human and murine cells. Eur J Hum Genet 21:948-956

Saeki Y, Breakefield XO, Chiocca EA (2003) Improved HSV-1 amplicon packaging system using ICP27-deleted, oversized HSV-1 BAC DNA. Methods Mol Med 76:51-60

Wade-Martins R, Smith ER, Tyminski E, Chiocca EA, Saeki Y (2001) An infectious transfer and expression system for genomic DNA loci in human and mouse cells. Nat Biotechnol 19:1067-1070 